\title{
Pediatría
}

http://www.revistapediatria.org/

Regional Bogotá

DOI: https://doi.org/10.14295/rp.v53i1.172

Carta al editor

\section{Rizotomía dorsal selectiva para manejar la espasticidad en niños con parálisis cerebral}

\section{Selective dorsal rhizotomy to manage spasticity in children with cerebral palsy}

\author{
Sandra Martínez-Pizarro ${ }^{a}$ \\ a Centro de salud de Granada. España.E-mail : mpsandrita@hotmail.com
}

\section{Sr. Editor:}

La parálisis cerebral (PC) es la discapacidad física infantil más frecuente. Tradicionalmente, los niños con hipertonía que no eran ambulatorios fueron considerados candidatos para la terapia de baclofeno intratecal para mitigar las molestias, mientras que la rizotomía dorsal selectiva (SDR) se reservaba para niños ambulatorios para mejorar la marcha. En la actualidad, el tratamiento quirúrgico de SDR está cobrando importancia debido a que se está comprobando que ofrece a todos los niños afectados buenas oportunidades para mejorar la calidad de vida (1).

En el estudio de Velnar T. et ál. (2), realizado en 2019 en Eslovenia, se presenta el caso exitoso de un niño de 15 años que fue tratado quirúrgicamente por espasticidad como resultado de PC. Se realizó laminotomía a nivel L1 y se aislaron las raíces nerviosas L1 a S1, entonces, se hizo la SDR. Los resultados mostraron que la SDR es una técnica quirúrgica eficiente para reducir la espasticidad. Requiere neuromonitorización meticulosa durante la operación y rehabilitación infantil continua con seguimiento a largo plazo. No obstante, el resultado del tratamiento de la SDR es duradero y los efectos secundarios con una cirugía cuidadosa son mínimos.

Los autores del estudio de Graham D. et ál. (3), realizado en 2018 en Australia, indican que la SDR es un procedimiento neuroquirúrgico que reduce permanentemente la espasticidad de las extremidades inferiores en niños con PC dipléjica espástica. La técnica actual se realiza a través de una laminectomía de nivel único a nivel del cono y, con la ayuda de la electromiografía intraoperatoria, se permite la división selectiva de las raíces nerviosas lumbosacras aferentes. En niños la reducción de la espasticidad tiene efectos positivos durante su crecimiento. La SDR se asocia con complicaciones mínimas y buenos resultados a largo plazo.
En el estudio de Kainz H. et ál. (4), realizado en 2019 en Bélgica, se analiza la influencia de la SDR en las fuerzas musculares durante la marcha. Se recogieron datos de 25 niños con PC espástica y 10 participantes con desarrollo típico. Los resultados mostraron que la SDR mejora las fuerzas musculares dinámicas en niños con PC y conduce a una menor patología de la marcha, como se muestra en la mejora de la cinemática articular y la cinética articular.

En el estudio de Jeffery SMT. et ál. (5), realizado en 2019 en Reino Unido, se evalúan los resultados quirúrgicos y las complicaciones producto de la SDR. Los niveles preoperatorios del sistema de clasificación de la función motora gruesa en 150 pacientes fueron II (35\%), III (65\%) y IV (1\%). La mediana de edad fue de 6 años. No hubo muertes, fugas de líquido cefalorraquídeo, reingresos, déficits motores o del esfínter. El dolor neuropático postoperatorio se informó en un 5,3\% y los síntomas sensoriales en un 8,7\%. Por lo tanto, la SDR es un procedimiento seguro con baja morbilidad quirúrgica. Este estudio complementa la creciente base de evidencia en apoyo de la SDR para la diplejía espástica y debería ayudar a informar las decisiones al considerar las opciones de tratamiento.

Si se analizan críticamente los resultados obtenidos en los estudios científicos descritos anteriormente realizados en los últimos años en diversos países (Estados Unidos, Eslovenia, Australia, Bélgica, Reino Unido), se puede observar el potencial que tiene el tratamiento de la SDR en niños con PC.

Estos estudios muestran a la SDR como un tratamiento efectivo, bien tolerado, con un buen perfil de seguridad y con unos efectos adversos leves y escasos; aunque cabe destacar que es importante continuar la investigación dentro de este ámbito para valorar los efectos de la SDR en estudios con mayor número de muestra, el seguimiento a largo plazo y su posible efecto sinérgico con otras terapias. 
A raíz de estos resultados es fundamental trasmitir esta información a los profesionales sanitarios que trabajan a diario con este tipo de pacientes. De esta manera, se podrá ofrecer a los pacientes los mejores cuidados y tratamientos basados en las últimas evidencias científicas demostradas.

\section{REFERENCIAS}

1. Davidson B, Schoen N, Sedighim S, Haldenby R, Dalziel B, Breitbart $\mathrm{S}$, et al. Intrathecal baclofen versus selective dorsal rhizotomy for children with cerebral palsy who are nonambulant: a systematic review. J Neurosurg Pediatr. 2019; Oct 18:1-9.
2. Velnar T, Spazzapan P, Rodi Z, Kos N, Bosnjak R. Selective dorsal rhizotomy in cerebral palsy spasticity - a newly established operative technique in Slovenia: A case report and review of literature. World J Clin Cases. 2019; 7(10):1133-1141.

3. Graham D, Aquilina K, Mankad K, Wimalasundera N. Selective dorsal rhizotomy: current state of practice and the role of imaging. Quant Imaging Med Surg. 2018; 8(2):209-218

4. Kainz H, Hoang H, Pitto L, Wesseling M, Van Rossom S, Van Campenhout A, et al. Selective dorsal rhizotomy improves muscle forces during walking in children with spastic cerebral palsy. Clin Biomech (Bristol, Avon). 2019; 65:26-33.

5. Jeffery SMT, Markia B, Pople IK, Aquilina K, Smith J, Mohamed AZ, et al. Surgical Outcomes of Single-Level Bilateral Selective Dorsal Rhizotomy for Spastic Diplegia in 150 Consecutive Patients. World Neurosurg. 2019 May; 125: e60-e66. 